\title{
CONFlicto y PAZ EN COLOMBia: UNA PERSPECTIVA DESDE LOS INDICADORES SOCIALES
}

\author{
CONFLICT AND PEACE IN COLOMBIA: \\ A PERSPECTIVE FROM THE SOCIAL INDICATORS
}

Por: Liliana Mendoza Ramos

inistradora de emis (2006); MBA en ción y Adminis-

on de Empresas

7); Candidato a ster en Desarrollo (2015). Expe-

cia en Docencia

ersitaria de nueve

Docente Cate-

ica Universidad artagena (pregraposgrados desde

). Asesora Ex-

de la Secretaría

cienda Distrital

2011). Docente

empo Completo e

stigadora Funda-

Universitaria Tec-

gico Comfenalco le 2013).

Recibido: 31 de marzo de 2015 - Aprobado: 10 de abril de 2015

\section{RESUMEN}

El presente estudio pretende elaborar un análisis cualitativo acerca de las variables que afectarán un eventual proceso de paz en términos de sostenibilidad. Para efectos del análisis se aborda la dinámica intrafamiliar (afrontamiento familiar) como variable interna y los indicadores sociales en Colombia (calidad de vida y pobreza), como variables externas al individuo. Inicia con un recorrido de investigaciones preliminares, en busca de una explicación histórico-social del contexto, basado en el comportamiento de los indicadores seleccionados.

Se concluye que un cambio de "Estado en conflicto" a "Estado de paz", reposa sobre la convergencia de indicadores que van más allá de un acuerdo político en La Habana que signifique una reestructuración económica y política institucionales y que por tanto su sostenibilidad depende de la comprensión de la dinámica familiar, la instauración de una nueva cultura política, y el aseguramiento de procesos de reinserción equitativos y dignos.

Palabras claves: Afrontamiento familiar, conflicto, paz, reinserción, sostenibilidad, variables sociales.

\begin{abstract}
This study aims to develop a qualitative analysis of the variables that will affect an eventual peace process in terms of sustainability. For purposes of analyzing domestic dynamics (family coping) as internal variable and social indicators in Colombia (quality of life and poverty), and external to the individual variables are addressed. Begins with a brief of preliminary investigations, in search of a socio-historical context, explanation based on the behavior of the selected indicators.

We conclude that a change of "Conflicted" to "state of peace" rests on the convergence of indicators that go beyond a political agreement in Havana that means an economic institutional political restructuring and therefore its sustainability depends understanding of family dynamics, the establishment of a new political culture, and ensuring equitable and dignified processes reintegration.
\end{abstract}

Keywords: Family coping, conflict, peace, reintegration, sustainability, social variable. 


\section{De la paz y el conflicto en Colombia}

ucho se ha escrito de la situación social y política de Colombia de
los últimos 50 años. El conflicto interno que involucra los grupos
alzados en armas y el Estado como principales protagonistas, es una de las fuentes más utilizadas en los análisis. Los relatos e investigaciones refieren desde el presunto origen de la guerra en Colombia hasta el análisis en perspectiva de un eventual tratado en La Habana. El conflicto ha sido explicado desde su rudimento filosófico, hasta llegar incluso, al análisis de la mutación de actuaciones en un contexto globalizado que ha obligado a sus actores a modificar las condiciones, los escenarios y otros fenómenos, que han coadyuvado a la regionalización del conflicto en el país (Mantilla, 2009).

Para Valencia, 2007, entre los años 1999 y 2006, la connivencia entre las dirigencias regionales, el poder político representado en el Estado, los paramilitares y el narcotráfico, quienes coincidían en la defensa de intereses particulares contra la posibilidad de paz entre el Estado-guerrilla, dio paso a la expansión y fortalecimiento del conflicto colombiano, cambiando el mapa político del país en función de los objetivos particulares de cada uno. En todo caso, el conflicto se ha configurado y reconfigurado en orden a unas variables de insatisfacción social, expresadas a través de la materialización o uso de la fuerza, que antepone el bienestar individual al colectivo. Teniendo en cuenta que Rosanvallón, 1995 (citado en Furlani, 2009), define el Estado Moderno como "...Estado protector y su existencia adquiere sentido como tal, si y sólo sí garantice, y defiende los derechos individuales del hombre" y que para Acemoglu y Robinson (2012), el entendimiento de variables como el poder, la pobreza y la prosperidad económica, obedece a la forma cómo se concibieron las diferentes sociedades en el marco de su historia, circunscritas a la voluntad política, es posible inferir que el Estado no sólo es garante de la seguridad de su nación, sino artífice principal del conflicto y de la paz; esta apreciación convierte a sus representantes en los principales autores y coautores de los conflictos, de sus causas, al igual que la implementación de las soluciones en un territorio democrático como el colombiano.

Generalmente los análisis de las causas y consecuencias del conflicto colombiano se abordan desde el aspecto político o económico, con lo cual se ha menospreciado una causa social que por ingenua que parezca, impacta en los índices de inequidad, violencia, estabilidad política e incluso, el desarrollo humano; se refiere en este caso a la dinámica familiar. 
Debido a las limitaciones que presentan las mediciones económicas de la realidad social, y su impacto directo en el diseño de políticas públicas concebidas para avanzar en la mitigación de la pobreza y la desigualdad, surge la concepción del Índice de Desarrollo Humano (IDH) como una alternativa de inclusión y cambio en el análisis de las condiciones estructurales de una sociedad. Este índice involucra aspectos como la posibilidad de mayor esperanza de vida, menores riesgos de muerte durante la niñez, superación de la desnutrición y el analfabetismo, como factores definitivos en la medición de la calidad de vida de los individuos en una sociedad. Ahora bien, si se entiende que una sociedad es constituida por un grupo de familias, y que a su vez, cada familia es constituida por un grupo de individuos, que al igual que un sistema abierto, está inserto en un entorno que le condiciona física, biológica, social y psicológicamente para adaptarse y sobrevivir a las perturbaciones y cambios de esos ambientes, se está frente a una relación interna-externa, donde necesariamente confluyen actitudes, aptitudes, valores, conocimiento individual y colectivo en el desarrollo del individuo y por ende, el desarrollo de la sociedad. En otros términos, el estado de bienestar interno (individual) impacta el estado de bienestar externo (colectivo).

En este sentido, los procesos de conflicto en las sociedades se derivan inicialmente de un estado interno individual que en primer lugar, hace eco en el estado de la familia, y que por lo tanto, si no se cuenta con herramientas psico-sociales que orienten un comportamiento adecuado, las consecuencias serán expresadas en los diferentes ambientes, esto es, en la comunidad o sociedad en que ocurran. Esta es la razón que ampara considerar una profundización en el estudio de la dinámica de las familias colombianas, no solo como principal aliado en procesos de posconflicto, sino como estrategia fundamental en la prevención de futuros conflictos. Uno de los frentes de estudio de la dinámica familiar, es la forma como sus integrantes enfrentan los problemas, cambios intempestivos o retos; este conocimiento insta a la intervención de conflictos familiares con herramientas adecuadas, bajo programas adecuados. En la práctica, significa conocer los síntomas para curar o prevenir las causas y no solamente tratar las consecuencias. Así, para la construcción sostenible de la paz, tan importante como las medidas políticas y económicas, resulta la definición o entendimiento de estructuras familiares que facilitan la inserción de los individuos en una cultura social renovada, que afiance procesos valorativos del bienestar colectivo, más allá de un eventual acuerdo entre el Estado y los grupos alzados en armas, que en términos reales es apenas la punta del iceberg ${ }^{1}$. 


\section{Construcción de paz y prevención del conflicto desde la familia}

En la Constitución colombiana de 1991, en su Capítulo 2, Artículo 42, la familia está definida como:

“...el núcleo fundamental de la sociedad... El Estado y la sociedad garantizan la protección integral de la familia. La ley podrá determinar el patrimonio familiar inalienable e inembargable. La honra, la dignidad y la intimidad de la familia son inviolables. Las relaciones familiares se basan en la igualdad de derechos y deberes de la pareja y en el respeto recíproco entre todos sus integrantes" (CPdC, p.6).

Lo anterior respalda la responsabilidad del Estado frente a las demandas de la familia como unidad social constitutiva de una nación.

Históricamente el estudio del individuo y su comportamiento, las desviaciones y patologías presentes en la vida de una persona (aquellas que no están en consonancia con la satisfacción en un individuo) u otros relacionados con su funcionamiento psicológico, y el proceso de maduración de los mismos, han sido focos de atención para las Ciencias Sociales en disciplinas como la Antropología, Pedagogía y Sociología; por ello, los estudios alrededor de las estrategias familiares aplicadas en momentos de crisis o estrés responden a la necesidad de conocer los sabidos esfuerzos cognitivos, conductuales que realizan las familias, sean estas funcionales o no, independientemente del número de individuos que la conforman y cuáles sean sus etapas de crecimiento.

Por otra parte, las personas, una vez interpretan cognitivamente las circunstancias que caracterizan su entorno, asumen una posición frente a ellas, lo cual se constituye en una respuesta que refleja varios estadios o niveles en su selección. Así, si los hechos suponen una amenaza para la integridad física o psicológica del individuo, este elabora una serie de alarmas que sugieren el intento por recuperar su equilibrio (Pérez, 2013), por lo cual elabora estrategias correctas o incorrectas que desarrollan modos de ser, de moverse y de convivir con la realidad que ha captado; estas reacciones son llamadas estrategias de afrontamiento.

A partir del surgimiento de la noción de resiliencia ${ }^{2}$ ha crecido el interés por conocer las capacidades que pueden estar presentes en cada uno de los miembros de la familia. La observación del desarrollo de la vida de seres humanos en su conjunto, amplía la perspectiva para describir el nivel de relacionamiento individual y grupal. Algunas experiencias consideradas 
adversas o importantes y que son acaecidas en el núcleo familiar, son aquellas que implican carencias, abusos, sobreprotección, descalificación, negligencia e ineficacia parental de quienes lideran los grupos sociales (Wolin y Wolin, 1993, citado en Ravazzola, 2001), la falta o inexistencia de gratificaciones, la falta de oportunidades, participación activa y positiva, las circunstancias de pobreza y marginación, son algunas otras experiencias adversas presentes en la familia, que aunadas a la exclusión social generan un concierto de actitudes de los individuos que la integran hacia su entorno.

De factores como la ansiedad, el desafío, la expectativa, e incluso la percepción de la propia incapacidad de responder ante el agente estresor interno o externo, emergen un conjunto de estrategias de afrontamiento, las cuales -a diferencia de los mecanismos de defensa que intentan proteger al individuo de esos eventos- , las define Kramer (2010) como abiertas o encubiertas, cuya finalidad es reducir o eliminar los estadios de amenaza, angustia o estrés psicológico; en consecuencia "el afrontamiento se define como los mecanismos provocados por procesos secundarios del pensamiento ${ }^{3}$ que permiten la elección deliberada, flexible y eficiente en el individuo y afectan su expresión" (Haan, 1977, 1982, citado en Kramer, 2010, pág 208).

El afrontamiento es definido por Lazarus y Folkman (1986) como los esfuerzos cognitivos y de comportamiento de la persona para reducir, minimizar, dominar o tolerar las exigencias internas y externas acerca de la transacción que representa la relación persona-ambiente, que se perciben como una amenaza potencial al bienestar y la regulación de las emociones. Esta respuesta puede estar centrada en una respuesta agresiva en un esfuerzo por superar o alterar la situación, también puede incluir un comportamiento frío, racional y deliberado que implique distanciamiento, auto-control, búsqueda de apoyo social o se asuma un comportamiento de evitación o escape que acepta la responsabilidad y la reevaluación positiva. Para García y Rodríguez (2005), el afrontamiento familiar se trata de los esfuerzos realizados y los recursos utilizados en la solución de una situación estresante, que en un circuito lógico proporcionan tranquilidad, compensación o equilibrio, lo que permite al individuo o la familia redefinir su rol, modificar sus metas, adaptarse o aprender de la experiencia.

Las respuestas pueden presentar un enfoque basado en el problema o en las emociones, por lo que McCubbin y colaboradores (1981), elaboran cinco tipos principales de recursos: Obtención de Apoyo Social, Reestructuración del Problema, Búsqueda de Apoyo Espiritual, Movilización Familiar para Obtener y Aceptar Ayuda y Evaluación Pasiva. La búsqueda de apoyo espiritual se 
manifiesta con decisiones como asistir a eventos religiosos, participando de estas actividades o buscando consejo en un sacerdote o pastor y teniendo fe en Dios; la movilización familiar para obtener y aceptar ayuda se refiere a las habilidades de los integrantes para buscar recursos en la comunidad y aceptar ayuda de otros; la evaluación pasiva es puesta en práctica cuando los miembros (o alguno de ellos) de la familia optan por disminuir la sensación de estrés participando de actividades que minimicen el problema, como ver televisión o acudir a juegos de suerte (Mendoza-Ramos, 2015).

Para el caso de las familias colombianas, es innegable el efecto que han causado las circunstancias de guerra, narcotráfico y paramilitarismo en el núcleo familiar. El solo fenómeno del desplazamiento, que ha sido una característica de comunidades enteras en el territorio nacional y que se considera como una característica de sociedades en conflicto cuando se produce bajo circunstancias de amenaza o en ambientes forzados (Newman y Selm, 2003), ha victimizado a familias completas, obligándolas a reconstruir por completo sus vidas, en cuyo proceso, deben renovar su forma de subsistencia. Las características particulares y contextuales de Colombia han dado paso al estudio transcultural de las estrategias por las que optan las familias en situación de estrés.

Debido a los efectos que ejercen las circunstancias en la elaboración de estrategias de afrontamiento de las familias colombianas, en especial aquellas que han sido sometidas o victimizadas por la situación de conflicto, las investigaciones en el nivel nacional han intentado comprender la dinámica interna y externa que asumen los individuos que pertenecen a estos núcleos familiares. En una investigación de Meléndez, 2010, halló que en familias víctimas de violencia intrafamiliar y desmovilizadas del conflicto armado en Colombia, recurren con mayor frecuencia a la reestructuración, esto es, que deciden cambiar el panorama a la situación problema y generalmente acuden a la evitación o distracción en espacios fuera del núcleo familiar, lo que expresaron les permite reevaluar de manera positiva el problema. Turizo, 2012, pudo determinar que los familiares de jóvenes desplazados que se radicaron en la ciudad de Barranquilla, utilizan el apoyo familiar como recursos para afrontar y controlar las condiciones aversivas del entorno de tal forma que la familia se convierte en un apoyo emocional y fuente de soporte económico, no obstante en algunas situaciones puntuales, prefieren que sus problemas sean resueltos al interior del propio núcleo familiar.

En Bogotá, fue estudiada la forma como las familias con hijos adolescentes afrontan una crisis causada por el desempleo, quienes reflejaron una preferencia hacia la estrategia de reestructuración del problema. Sin embargo, los sujetos 
estudiados se resisten particularmente a vivir separados de sus hijos o disolver el vínculo conyugal, aun cuando la percepción de crisis sugiera una actitud de angustia, pues consideran que la posibilidad de mantenerse unidos proporcionó una sensación de fortaleza, lo cual puede estar amparado en factores socioculturales e idiosincrático (Martín, Fajardo, Gutiérrez y Palma, 2007). Las familias que son víctimas de secuestro extorsivo económico emplean diferentes estrategias dependiendo de las demandas que surgen en el proceso. Para el caso de familias sometidas al secuestro de uno de sus miembros se manifiestan diferentes estados que van desde el secuestro hasta las promesas y las amenazas durante la negociación, por lo tanto, algunas veces se exige manejar las tensiones originadas por la situación, mirar con cierto optimismo que volverán a ver a su familiar; y otras veces se les exige eliminar la carga negativa del trauma y redefinir las circunstancia; mientras para aquellas familias sometidas al secuestro de uno de sus miembros, mostraron que el afrontamiento centrado en el manejo del problema puede favorecer en estas circunstancias.

En otras latitudes los hallazgos son fundamentalmente similares en circunstancias parecidas, por ejemplo Ruano y Serra (2000) describen estrategias de afrontamiento que usan los padres en la Comunidad de Valencia de España, con hijos adolescentes (entre 12 y 19 años de edad) a fin de contrastar cuáles resultan ser más útiles. Los 386 padres sujetos al test F-COPES, consideran la estrategia de reestructuración y obtención de apoyo social como las más útiles frente al resto, al igual que Cadavid (2007) y por otra parte Jiménez, Amarís y Valle (2012) lo hallaron en familias colombianas. En Denver USA, un estudio de afrontamiento personal y familiar en relación con la salud mental de los jóvenes en 82 adolescentes, muestra que la evitación y la negación no sugieren perjuicio para los adolescentes varones y niñas en el corto plazo, sin embargo en el largo plazo, este tipo de estrategias empeora los conflictos, en contextos de pobreza, que se considera un factor que exacerba el estrés en detrimento de la salud mental de los adolescentes por lo que los conflictos al interior de la familia resultan más difícil de manejar en altos niveles de estrés generados por situación de pobreza, y es asociado a ella significativamente la angustia psicológica, con lo cual el mejoramiento de las habilidades de afrontamiento puede disminuir los efectos negativos del estrés (De Carlo y Wadsworth, 2009). En investigación posterior de los mismos autores, los resultados sugieren que si se establece el uso de estrategias de afrontamiento de control primario y secundario, el beneficio será mayor para los niños y las familias en cuanto a sus respuestas a los agentes estresores ya que estas respuestas influyen en el desarrollo de las habilidades en los niños, 
lo que es pertinente con la implementación de programas que enseñen a los niños estrategias de afrontamiento activas y que las de evitación y negación sean usadas con menos frecuencia.

Los estudios encontrados registran las más diversas líneas del conocimiento, pero todas confluyen en la dinámica familiar, sus ajustes y demandas. Por ejemplo, la descripción de estrategias más usadas por parejas en situación de desempleo (Martín et al, 2007); en jóvenes desplazados (Turizo, 2012); en víctimas de secuestro extorsivo (Navia, 2008), recrea las respuestas del núcleo en situaciones imprevistas o cambios de estado, en todo caso, en sucesos que no podemos evitar que acontezcan. Hallar posibles relaciones entre la violencia intrafamiliar y las estrategias utilizadas en familias desmovilizadas (Meléndez, 2010) coadyuva a la posibilidad de establecer intervenciones para la reparación mental, emocional, psicológica y relacional de los individuos en tal situación. En este grupo de familias las estrategias más frecuentes encontradas son reestructuración, apoyo social, apoyo familiar y en algunos casos evitación.

Otros ejemplos como el estudio de familias con pacientes enfermos mentales (Grey, 2006; Caqueo y Lemos, 2008; Kartalova-O'Doherty y Tedstone, 2008; Ruano y Serra, 2000; Gerson, Wong, Davidson, Malaspina et al, 2011; Eaton, Davis, Hammond, Condon y McGee 2011); con familiares con trastorno alimentario - anorexia (Cunha, Relvas y Soares 2009); en contextos de pobreza y/o traumáticos (Wadsworth, 2008; Kiser et al, 2010; De Carlo et al, 2012;) y jóvenes con intento de suicidio (Fidan, Ceyhun y Kirpinar, 2011), abordan problemas de salud física o mental, en busca de bases para fundamentar tratamientos efectivos, pues en estos casos la estrategia menos utilizada es el apoyo familiar.

Las investigaciones que se aluden en el presente análisis, sin pretender ser exhaustivos, se han realizado en el país, con resultados similares de aquellas hechas en otras latitudes. Esto permite inferir que en situaciones de estrés, las familias utilizan estrategias diferentes que les orienten hacia una salida racional del problema. En términos generales, la forma como las familias enfrentan situaciones que les acontecen, se adaptan o se reestructuren, manejan una relación simbiótica con otros factores como los emocionales, psicológicos y conductuales que se expresan o tienen impacto en eventos o agentes externos más próximos, por ejemplo en quienes les rodean, sean amigos, vecinos o familiares. Se infiere que la identificación de estas formas de respuestas puede conducir eventualmente a la intervención y/o prevención de conflictos al interior de las familias y evitar el efecto "rebote" en su entorno, 
esto es, incidir sobre la raíz de las causas que originan los problemas de adaptación, tolerancia y violencia.

Por diferentes que sean sus perspectivas, las investigaciones confluyen en que los elementos singulares del afrontamiento en las familias son significativo en la construcción de nuevos cánones de comportamiento; o como lo expresan Azevedo y Bouillon (2009), los antecedentes generacionales influyen cognitivamente en la acumulación de capital humano.

\section{Los verdaderos enemigos de la paz sostenible}

i bien el estudio sobre la dinámica familiar nos induce hacia un diagnóstico de la salud intrafamiliar ${ }^{4}$, cuyos síntomas pueden ser tratados $\checkmark$ desde la intervención psico-social, al otro lado de la ecuación se encuentran agentes externos, que de una u otra forma motivan los cambios estructurales para el fortalecimiento y sostenibilidad de ese bienestar; en aquí donde intervienen las variables sociales, económicas y políticas cuyas decisiones provienen del Estado, y este orden, garantizan el bienestar de cada nación. La perspectiva de análisis en este trabajo, describe aquellos factores externos que dependen de la gestión estatal, basándose en las estadísticas de los indicadores socio-económicos, que se considera intervienen en la puesta en escena de una paz sostenible para el territorio nacional. Posteriormente se realiza un análisis cualitativo de las variables familia-estado, en búsqueda de posibles alternativas que garanticen, en menor o mayor grado, la renovación social de los colombianos.

\section{- Indicadores sociales ${ }^{5}$}

Tradicionalmente, los indicadores sociales nos dan una apreciación estadística del estado de bienestar de una comunidad o población, como referencia de aquellos que fundamentalmente impactan en los individuos, y que se consideran que mientras más negativo sea su comportamiento, menor será el alcance de estándares sociales dignos. A continuación y para efectos del análisis del presente documento, se presentan algunos de ellos.

\section{- Calidad de vida.}

Caracteriza la población en los aspectos que involucra el bienestar de los hogares. Cuantifica variables relacionadas con la vivienda, educación, salud, cuidado de los niños, fuerza de trabajo, gastos e ingresos. En la construcción del presente análisis y con el objeto de acotar el ejercicio investigativo, se tomarán las tres primeras. 
Las condiciones materiales precarias de las viviendas en una sociedad, son consideradas una forma de exclusión social, al igual que el poco o nulo acceso al empleo, educación y servicios de salud (Irigoyen-Coria, Gómez-Clavelina, Terán y Ponce-Rosas, 1999). De acuerdo con el DANE -Departamento Administrativo Nacional de Estadística - en promedio, en 2013 el 77.4\% de los colombianos tiene acceso a los servicios públicos domiciliarios, siendo el de energía eléctrica el de mayor cobertura nacional $(98,2 \%)$, seguido de la tenencia de un teléfono celular (94,7\%). En su orden, el acceso al sistema de salud, servicio de alcantarillado y gas natural reportaron comportamiento similares, en un 91.3\%, 75.9\% y 58.9\%. Si bien el indicador muestra una tasa de cobertura, en términos de calidad (aspecto cualitativo) no es posible hacer inferencia desde esta mirada.

Por su parte la educación representa un proceso complejo, multidimensional y multidireccional en el que se pretende definir características culturales, morales, éticas, estéticas en los individuos con el propósito de transformarle cognitiva, emocional y socialmente de tal forma que adquieran la capacidad de tomar decisiones personales y en su conjunto con integralidad y suficiencia. En términos sociales, esta es otra de las variables relacionadas con la calidad de vida, la cual según León (2007) busca la perfección del ser humano y le da un cierto grado de libertad.

En Colombia el acceso al sistema educativo es un derecho fundamental de las personas. En la década de los 80 la preocupación en el país estaba centrada en la tasa de cobertura de la educación, sin embargo en épocas recientes el énfasis se encuentra en la calidad. Si bien este concepto es relativo por cuanto existen factores históricos que determinan las expectativas colectivas, los elementos que convergen en la calidad educativa están íntimamente relacionadas con la finalidad, su eficiencia y costo (Castaño-Duque y García Serna, 2012). De esta forma, se aprecia no solo la mayor cobertura posible (Cuadro 1. Matrícula por nivel educativo), sino que la estructura de formación sea comparable con los estándares nacionales e internacionales.

Cuadro 1. Matrícula por nivel educativo en Colombia

\begin{tabular}{|l|c|c|c|}
\hline \multicolumn{1}{|c|}{ NIVEL EDUCATIVO } & Matrícula 2012 & Matrícula 2013 & $\begin{array}{c}\text { Variación } \\
2012-2013\end{array}$ \\
\hline Preescolar & 1.107 .768 & 1.093 .675 & $-1,3 \%$ \\
\hline Básica Primaria & 4.659 .210 & 4.597 .486 & $-1,3 \%$ \\
\hline Básica Secundaria y media & 4.874 .265 & 4.849 .550 & $-0,5 \%$ \\
\hline Total matricula nacional & $\mathbf{1 0 . 6 4 1 . 2 4 3}$ & $\mathbf{1 0 . 5 4 0 . 7 1 1}$ & $\mathbf{- 0 , 9 \%}$ \\
\hline
\end{tabular}

FUENTE: Boletín Técnico DANE, septiembre 2014 
De los matriculados referidos en el cuadro 1, el 77,1\% fueron atendidos en establecimientos oficiales; en establecimientos no oficiales el 18,4\% y el 4,5\% corresponden a matrícula contratada ${ }^{6}$. El DANE reporta que el comportamiento anual del nivel de matriculados tiende a la baja desde el año 20097. A pesar de los evidentes avances en cobertura, la calidad del sistema educativo colombiano registra rangos inferiores en comparación con países latinoamericanos como Chile, México y Argentina ${ }^{8}$.

En el caso de la educación superior en el país, de acuerdo con las mediciones del Índice de Progreso de la Educación Superior (IPES) ${ }^{9}$ desarrollado por el Ministerio de Educación Superior, se establecen referentes regionales e instrumentos de medición para definir políticas basadas en resultados. Del año 2010 al 2013, la tasa de cobertura en materia de acceso a la educación superior pasó del 37.1\% al 45.5\%, con una tasa de deserción del 12.9\%; así mismo el índice de acceso a la educación superior aumentó del 29.9\% en 2012 a 32,4\% en 2013. Por otra parte, las exigencias hechas por el Ministerio de Educación Nacional (MEN) han motivado un mayor número de instituciones de educación superior a acreditarse y acreditarse en alta calidad, teniendo en cuenta los requisitos de cumplimiento para este nivel. El índice de calidad es medido a nivel nacional por los resultados de las pruebas Saber Pro, que sostuvo un incremento de un punto porcentual del 2013 con respecto al año anterior. Las mediciones del MEN registran diferencias significativas entre las regiones, siendo las regiones de Antioquia, Valle, Cafetera y Bogotá las regiones por encima del promedio y la Caribe, Oriental, Pacífica y Central con los índices más bajos en calidad ${ }^{10}$. Este indicador muestra una vez más la brecha de desigualdad en el país, en lo que corresponde a este factor. Sin embargo, no es solo la educación un elemento de movilidad y bienestar, sino que en la realización de los individuos intervienen de forma definitiva las estrategias activas de las familias y el apoyo de estas para asegurar modelos definidos en la estructura de la sociedad, que produzcan verdaderos cambios intergeneracionales (Vélez, 2014).

Desde otra arista de la calidad de vida se encuentra el acceso al sistema de salud y la calidad que este ofrece a los usuarios. En Colombia, aun cuando el acceso a la salud muestra un índice alto de personas adscritas a los regímenes ${ }^{11}$ (Gráfica 1), el colapso del sistema es evidente, en parte como consecuencia de los desfalcos importantes que han llevado al deterioro en la calidad de prestación del servicio. En el año 2012 se destapó el millonario desfalco al sistema por encima de los dos billones de pesos colombianos de acuerdo con declaraciones de la Contraloría General de la República ${ }^{12}$. 
La salud e integridad física son objetos jurídicos, pero no son independientes de la dignidad humana; la constitucionalidad colombiana ha reconocido la conexidad que tiene el sistema con los derechos fundamentales del ser humano. En este contexto, el derecho al acceso y calidad del sistema está fundamentada en los servicios recibidos del sistema y los planes obligatorios que deben proteger una vida digna ${ }^{13}$. Es común para un colombiano promedio recurrir a acciones legales válidas para defender su derecho a la salud, independientemente del régimen al que se encuentre afiliado; las redes tanto públicas como privadas de atención en salud se encuentran en crisis económica por los impagos procedentes

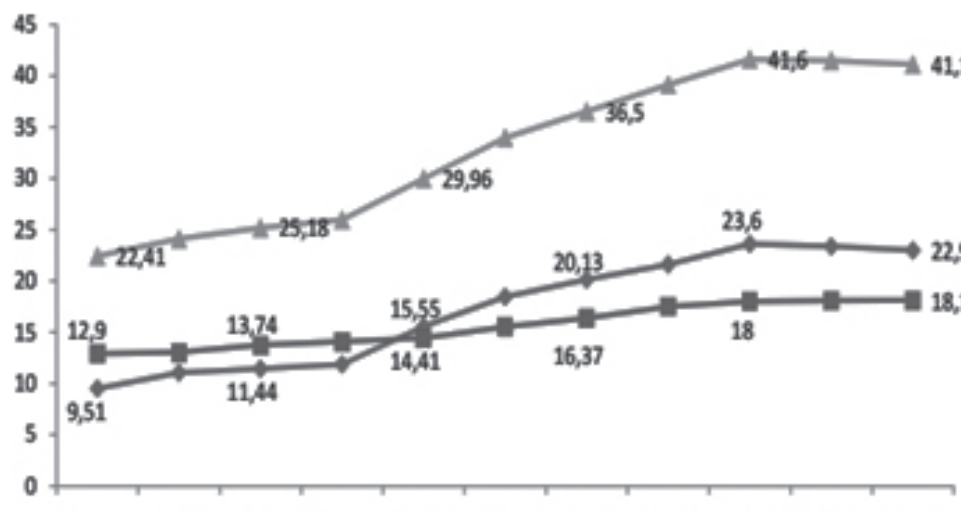

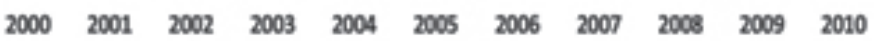
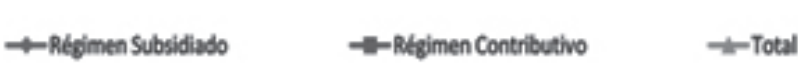

Gráfica 1. Afiliados al Sistema General de Seguridad Social e Colombia (millones) 2000-2010.

Fuente: Ministerio de la Protección Social. Informe abril de 2011. de las Empresas Promotoras de Saludo (EPS) a los prestadores del servicio (pág. 59). Al respecto Botero, Rendón y Herrera (2012) dicen:

“... en los casi veinte años de vigencia de la Ley 100, la salud en nuestro país terminó por convertirse en negocio económico, en donde la influencia del gobierno es desplazada por algunas empresas aseguradoras que son más bien intermediarias financieras. En este contexto, las EPS no asumen el rol de asegurar y administrar el riesgo como les corresponde." (pp. 183).

Por otro lado, diversos estudios científicos han podido determinar que la salud individual y familiar está influenciada por la dinámica familiar y los factores externos que les rodean, las estrategias de afrontamiento como la evitación influyen en la salud mental de sus miembros y está asociado con síntomas de altos niveles de estrés (De Carlo y Wadsworth, 2009). El sistema de salud colombiano dista aun de proporcionar las condiciones apropiadas para convertirse en instrumento de paz sostenible; es necesaria la reestructuración del sistema de tal forma que toda la articulación de sus actores esté basadas en la prevención de la enfermedad y en la promoción de la salud. Los individuos y las familias son el eje fundamental de cualquier propuesta conveniente para cambios importantes en esta variable.

\section{Pobreza monetaria y multidimensional.}

Esta variable se calcula desde los ingresos monetarios que perciben los hogares (pobreza monetaria) y la evaluación de cinco dimensiones que valoran las privaciones presentes en el hogar; el índice toma un valor de 0 a 100. Aquellos

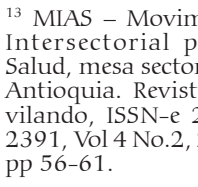
pp 56-61. 


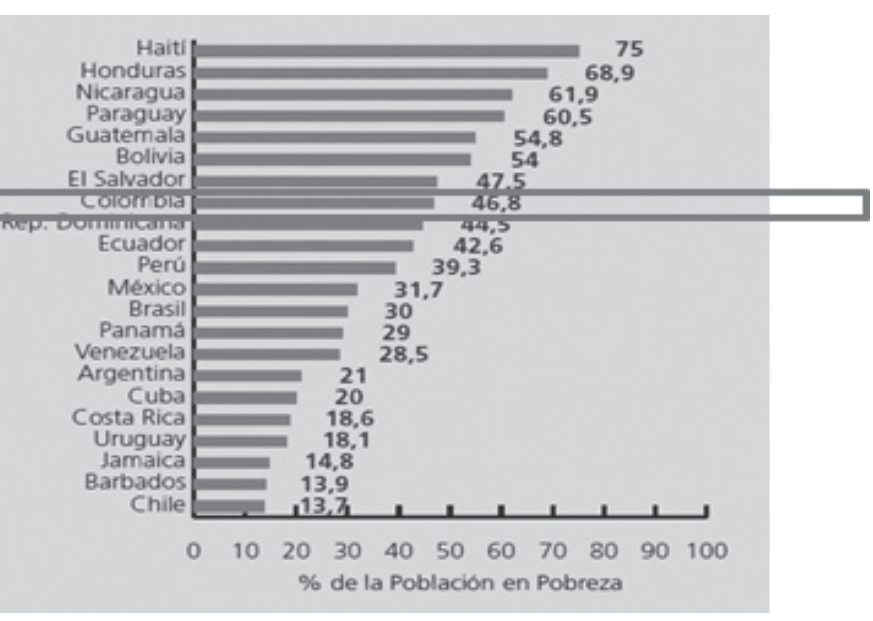

ífica 2. Tasas de pobreza en América Latina

te: Puryear y Malloy, (2009). Tomado de CEPAL, Panorama Social de América Latina, 2008. hogares que toman un porcentaje mayor o igual al $33 \%$, es considerado pobre. De acuerdo con el Boletín de Prensa del DANE, de marzo 21 de 2014, la pobreza en Colombia obtuvo las siguientes valoraciones:

- $\quad$ Para el 2013, el porcentaje de persona en pobreza fue del $30.6 \%$.

- $\quad$ El porcentaje de personas en pobreza extrema, $9.1 \%$.

- $\quad$ En términos de desigualdad el Gini ${ }^{14}$ registró un valor de 0.539 .

Las dimensiones de la pobreza (ingresos, educación, protección social y vivienda) mantienen una relación estrecha con problemas de mala salud sexual y reproductiva, trastornos psicológicos, angustia psicológica, disposición a ser fumador, así como una exposición frecuente a los riesgos que las inducen, lo cual proporciona una nueva visión sobre la consideración práctica de las dimensiones de la pobreza para la formulación de políticas públicas más eficaces (Oshio y Kan, 2014). Al respecto, Loignon, Hudon, Goulet et al, 2015, sostienen que los bajos salarios en una sociedad resultan en viviendas inadecuadas e inseguridad alimentaria $^{15}$; los pobres perciben barreras comunicativas por la impotencia y falta de recursos, lo que constituye de salida, una dificultad para el diálogo, la confianza y el compromiso. Los autores relacionan la pobreza como un determinante social de la salud; para Fidan et al (2011) la pobreza también mantiene una relación estrecha con la aparición de enfermedades, puesto que las familias en contexto de pobreza utilizan con menor frecuencia el apoyo familiar y esto les da menos opciones para la reevaluación positiva. Igualmente la inseguridad alimentaria tiene una relación evidente con la menor condición de bienestar en los hogares y consecuencias en la desnutrición, asociación con la contracción de infecciones y aumento del riesgo de muerte prematura, que invariablemente afectarán a la sociedad (Martínez-Rodríguez, García-Chong, Trujillo-Olivera y Noriero-Escalante, 2015). Así mismo, el grado de marginación e inequidad tiene una afectación en la salud de las familias que se refleja en la morbilidad, mortalidad, fecundación, educación, condiciones de la vivienda e ingresos (Irigoyen-Coria, et al, 1999), condiciones que exacerban los conflictos al interior de la familia, que en consecuencia adopta estrategias de evitación (Wadsworth, 2008).

A partir de la década anterior, Colombia ha venido trabajando en la modificación de las políticas macroeconómicas, con el propósito de consolidar 
el crecimiento económico y reducir la volatilidad de los indicadores globales. Sin embargo, los niveles de desigualdad en el ingreso per cápita permanecen y aun cuando algunos puntos porcentuales de la línea de pobreza han bajado, en segmentos específicos como la tercera edad, estos niveles siguen siendo superiores a los de la mayoría de economías de Latinoamérica (Gráfica 3).

En palabras de Azevedo y Bouillon, 2009, aspectos como la movilidad social está estrechamente relacionada con el grado de igualdad de oportunidades en un país, cuyas estimaciones para América Latina tienen un índice mucho más bajo que los países desarrollados, incluso aquellos que tienen más bajo nivel de movilidad (Estados Unidos

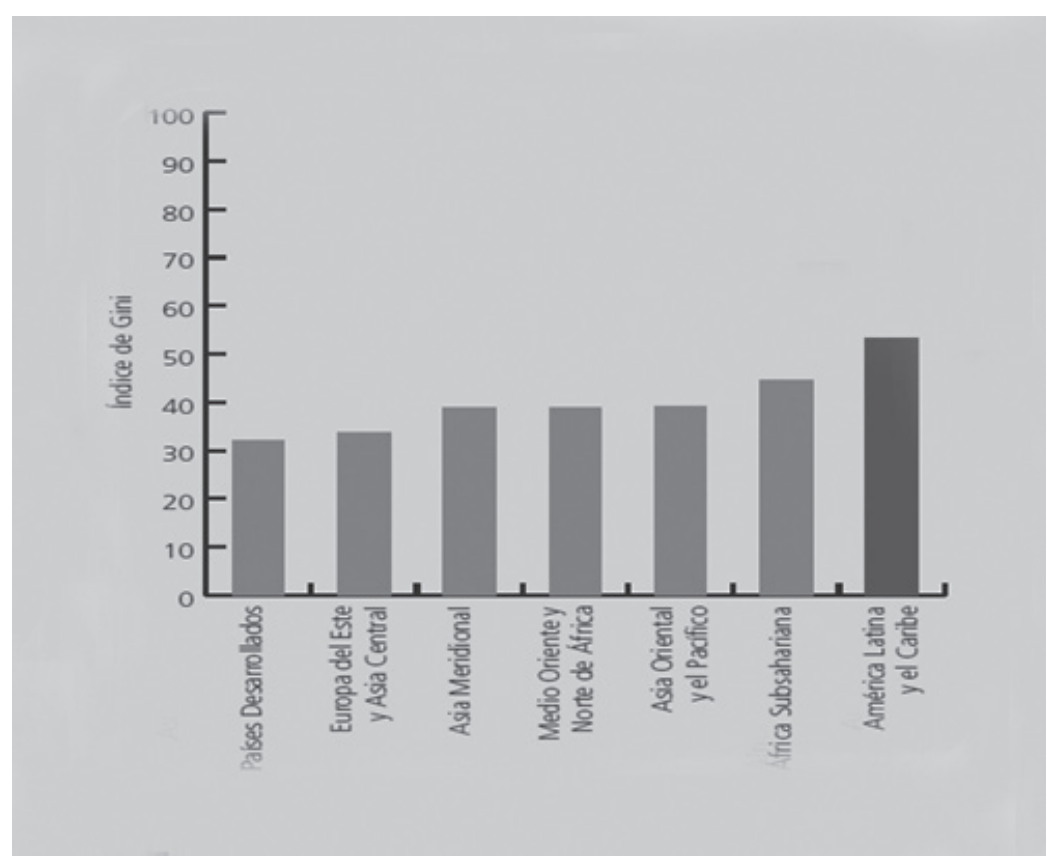

Gráfica 3. Niveles de desigualdad del ingreso por región.

Fuente: Puryear y Malloy, 2009. Pp. 2. y Reino Unido). El autor considera que herramientas de política pública como la meritocracia pueden constituirse en incentivos para la vinculación laboral, pero si no existe un factor de confianza en esas posibilidades pueden comportar indicadores de delincuencia o drogadicción; sin inversión en capital humano, no existen posibilidades para que los pobres se muevan hacia arriba, en términos generales, estos seguirán siendo pobres.

Uno de los factores que afectan la movilidad social ascendente en Colombia es el acceso limitado a la educación de los hogares pobres y el costo que representa para un colombiano llegar a niveles de formación superior, especialmente el nivel posgradual ${ }^{16}$. Si tomamos la definición de Ferreira y Gognoux, 2008, y Roeme, 1998 (citados en Galvis y Roca, 2014), el índice de movilidad social involucra variables como las circunstancias (como factor exógeno), el esfuerzo y la aleatoriedad (como factores endógenos), la desigualdad de oportunidad están condicionadas por la distribución del ingreso, sin embargo, esta distribución depende de la adopción de políticas económicas y sociales que permitan el apalancamiento de las clases más bajas hacia el escalonamiento social de mejores condiciones socio-económicas. En otras palabras, entre más equilibradas sean las distribuciones, menor será el índice de desigualdad social. De acuerdo con Galvis y Roca, 2014, para el caso colombiano, en las regiones más pobres de Colombia, la desigualdad en el ingreso (PIB per cápita) tiene una relación significativa en los índices de movilidad intergeneracional y que en esta se presentan diferencias significativas incluso de un grupo étnico a

${ }^{16}$ En la realizaci los Diálogos Regic sobre Educación movidos por el Co mavional de Educ Nacional de Educ
Superior, entre ma Superior, entre ma
junio de 2013 , la dad civil y la comu económica expre costos de la educ en Colombia. Un las peticiones está dedor que la educ superior públic: completamente g
ta y que existan ta y que existan n financiero estudi tasas más bajas, y subsidios que $\mathrm{g}$ ticen la perman de los estudiant la educación sup FUENTE: La voz regiones. CESU - Cc
Nacional de Educ Superior, julio 201

En 2013 solo el 3. los graduados en grado continúan programa de educ superior. FUE Graduados Colo para la educación. 
otro. En términos generales, el país presenta bajos índices de movilidad. Así las cosas, este indicador desarticula las bases para una solución sostenible del conflicto, siendo este factor un limitante para la superación de la pobreza.

En este sentido, los índices de pobreza en Colombia, se resisten a construir una fortaleza para un proceso de paz sostenible, máxime en un momento en que nos encontramos en la región más desigual del mundo (Gráfica 1. Tasas de pobreza en América Latina), por lo tanto el esfuerzo institucional para la superación de pobreza en el país deberá redoblarse en el diseño de estrategias estatales que permitan una mejor distribución del ingreso para crear condiciones más igualitarias en cuanto a los indicadores sociales se refiere.

Los indicadores estudiados datan de más años de historia colombiana que aquellos de existencia de cualquier fuerza armada revolucionaria o del narcotráfico. Los niveles de pobreza persisten con el paso del tiempo, a ello se unen los índices de desigualdad de ingresos por región; la calidad de vida en el país no presenta indicadores claros, si bien variables como la educación ha mejorado en accesibilidad, no lo ha hecho en calidad. Mendoza-Ramos (2013) alude a la responsabilidad del Estado en la conformación de políticas públicas dirigidas a las necesidades de la población, pues los problemas sociales devienen de la inconformidad de la sociedad. El ejercicio actual por conseguir un eventual tratado de paz para Colombia en La Habana (Cuba) es importante, sin embargo, no se deben desestimar los esfuerzos en los que incurrirá el país, si se da una mirada a la realidad económica, social y política que se sostiene, en especial porque las personas que hacen parte del conflicto armado no se pueden contemplar como unidades en el vacío. Los protagonistas de la guerra, una vez firmado el acuerdo, estarán abocados a la inserción en primer orden, a una familia, si la tienen. De este fenómeno se derivan las acciones que asumirá internamente esa familia al acoger nuevamente un integrante, adaptarse a las nuevas condiciones y asumir una estrategia de reestructuración positiva o una evitación ante las circunstancias. En segundo lugar, la inserción social y laboral que asegure la estabilidad de cada individuo en su rol como ciudadano. Ambas situaciones (interna y eterna) necesitan el acompañamiento y la observancia de su progreso.

Es indiscutible que cada país posee su propio contexto y su propia historia. Sin embargo, es probable que a través de otras experiencias se puedan reconocer procesos con desempeño exitoso o poco fructífero. Casos centroamericanos como Guatemala ${ }^{17}$, cuyo gobierno firmó acuerdos de paz con la Unidad Revolucionaria Nacional Guatemalteca (URNG) a finales de 1996, después de 36 años de conflicto, ha experimentado un rearme bélico e ideológico de los 
grupos armados antiguos; se siguen presentando casos de violación de derechos humanos, revictimización, impunidad e ilegalidad, lo cual ha generado nuevos escenarios de guerra, un tejido social fragmentado y estigmatizado por la desigualdad e inseguridad de su población. La justicia transicional establecida como instrumento para el esclarecimiento de verdad y reparación de víctimas ha dado pocas reformas duraderas en el sistema judicial (Aldana, 2013). De manera similar, en El Salvador la inoperancia de las instituciones judiciales produjo después de los acuerdos de 1992 desconfianza en los procesos internos de reestructuración, en donde factores como la investigación limitada de los crímenes, la falta de independencia judicial y hechos de corrupción dieron paso a un control inexistente en la aplicación de la justicia (Garzón, 2003). En Guatemala, al igual que en el Salvador, los acuerdos de paz no se limitaron a perseguir el fin de la violencia, sino que sus objetivos institucionales fueron dirigidos a reformas estructurales y socioeconómicas que buscaran corregir las causas del conflicto; un informe de las causas del conflicto guatemalteco identificó una vinculación entre pobreza y violencia, no obstante los resultados del análisis de más de 20 años después de los acuerdos, coinciden en que la interpretación del conflicto debe servir de base para superar una cultura del enfrentamiento, sin embargo el enfoque de las sociedades pos-conflicto se enclava en resolver dilemas como la reclamación de la justicia, entrega de las víctimas de la guerra a sus familiares y explicación de la violencia sufrida (Garranzo y Gómez, 2006). En cualquier caso, las familias de un país están involucradas en la medida que alguno o algunos de sus miembros han sido víctimas o victimarios; ambos grupos buscan la reinterpretación del pasado como primer paso para la reconstrucción del futuro inmediato. Las respuestas de la familia a situaciones de crisis vuelven a jugar un papel importante.

La reestructuración económica, política y jurídica de un país no es suficiente para garantizar una verdadera paz sostenible. La dinámica familiar también cumple un papel preponderante en la reestructuración social; el comportamiento de sus miembros y el nivel de ajuste ante circunstancias externas componen un factor de apoyo en brotes de violencia; análisis realizados de zonas conflictivas reflejan que en las áreas donde se presentan mayores índices de violencia intrafamiliar, existe proliferación de violencia social (Cerda, Cerda y Rodríguez, 2009). La relación ambivalente sociedadestado y los resultados del equilibrio social dependen del funcionamiento adecuado de las instituciones y de las familias. Las variables sometidas a la presente reflexión, como calidad de vida, educación, salud y pobreza, no ofrecen por el momento, un panorama alentador que soporte una base viable en el largo plazo para el sostenimiento de un territorio de paz. Un mayor conocimiento del afrontamiento familiar podría dar nuevas herramientas para

17 Hernández,
(2011). Guatemal años después de lo: erdos de paz, la g continúa. Disponi http://tomalapal periodismohum $\mathrm{com} / 2011 / 12$ guatemala-15-a dos-de-paz-la-gu continua/ recup en junio 25/2015 
decantar las raíces de la violencia en un individuo y por tanto en una sociedad.

\section{Conclusiones y recomendaciones}

- En diferentes estudios nacionales e internacionales se ha evidenciado la importancia de los patrones formativos en la familia a temprana edad. La confrontación de los problemas y la búsqueda de soluciones, atribuyen a la familia un papel activo en la formación del ciudadano. Conocer aquellos factores que posibilitan una estructura comportamental sana, coadyuva a la consolidación de la paz en un concepto pluralista e integral, más allá de un acuerdo político o económico en La Habana.

- En los indicadores sociales tradicionales, debido a la medición para la que fueron diseñados, no se evidencia un flagelo que ha golpeado el núcleo de las familias colombianas, que va más allá de lo aparente y visible, como es la salud o el bienestar en las familias como consecuencia de la situación de guerra y desplazamiento. Por lo tanto, en el contexto del país, han generado al interior de su dinámica estrategias de afrontamiento, que dan cuenta de su capacidad de adaptarse, reestructurar los problemas o evitarlos. El conocimiento de estas características comportamentales dan herramientas científicas para el fomento de una cultura de paz desde la estructura básica funcional de la sociedad: la familia.

- El conflicto armado en Colombia tiene múltiples causas sociales, económicas, y políticas, que descansan sobre la incapacidad del Estado en el manejo de las instituciones cuyas actividades misionales se enfocan en salvaguardar la seguridad de la nación.

- Como todo fenómeno híbrido y mutante, la situación de conflicto colombiano posee varias causas, que deben ser identificadas. A nivel estatal se requieren acciones profundas que generen un cambio en las instituciones y en la concepción de políticas públicas que recuperen el orden y la dignidad de los nacionales, con el objeto de darle al eventual acuerdo de paz con las FARC la connotación de sostenible que garantice la generación de oportunidades reales en el proceso de reinserción social de los individuos.

- Los verdaderos enemigos de la paz sostenible proceden de las falencias en la gestión pública, instrumentalización de políticas efectivas, falta de gobernabilidad e institucionalidad, que impactan en el comportamiento de índices de pobreza extrema, desigualdad en la inversión y gasto de las regiones, calidad de vida en proporciones equilibradas para toda 
la nación, oportunidades de formación y cualificación, que permitan condiciones de vida dignas.

- En busca de garantizar un proceso de reinserción civil de los alzados en armas, en condiciones de igualdad, equidad y tolerancia se debe partir de la dinámica funcional de la familia, como es el abordaje de problemas y afrontamiento en situaciones de crisis o cambios. La salida, o en este caso, la adición de un miembro a la familia, es un acontecimiento que pasa por varios estadios y genera reacciones individuales en el resto de los integrantes. Será necesario la creación de herramientas estructurales que se ocupen del seguimiento o acompañamiento de las familias involucradas en los procesos de reinserción, cuyo estudio derive acciones dirigidas a la adopción de estrategias de reestructuración antes que estrategias de evaluación pasiva.

- La identificación de las respuestas de las familias ante eventos estresantes (afrontamiento familiar) o circunstancias propias de una sociedad pos-conflicto pueden coadyuvar en tres etapas fundamentales en la construcción de una paz sostenible: en la primera etapa de interpretación histórica del conflicto y perdón a los agresores; en una segunda etapa de adaptación a las nuevas condiciones (sea por desaparición y/o muerte de familiares o regreso de aquellos que hicieron parte de las filas de grupos alzados en armas) y en una tercera etapa de reconstrucción de una sociedad o impulso de nuevas generaciones no violentas, restándole significativamente el sentido histórico de esta para la obtención de mejores condiciones de vida.

- La experiencia de acuerdos de paz en el continente como Guatemala y El Salvador, pueden orientar hacia la construcción de procesos válidos para el caso colombiano, que eviten incurrir en equivocaciones jurídicas, económicas, políticas y sociales, en busca de una paz sostenible. Sus experiencias conducen a inferir que no solamente las reformas institucionales socio-económicas son las que garantizan el éxito de un proceso post-acuerdo.

- La reconstrucción social de Colombia no depende solamente de la eventual firma de un acuerdo de paz. En caso que este llegue a darse, es el inicio de todo un proceso complejo con dimensiones económicas, políticas y en especial, sociales. Incluso, habrá que determinar qué pasará con los niños y adolescentes que hacen parte de las filas de estos grupos y su reinserción en sus familias de origen. De lo contrario, se 
estará frente a un fenómeno con características de "ave fénix".

\section{BIBLIOGRAFÍA}

ACEMOGLU, D. \& ROBinSON, J. A. (2012). Por qué fracasan los países. Bogotá. Ed Planeta Colombiana S.A. 589 p.

ALDANA, R. (2013). A Reflection on transitional justice in Guatemala 15 years after the peace agreements En Victims of international crimes: An interdisciplinary discourse. Thorsten Bonacker \& Christoph Safferling (Ed). ISBN: 978-90-6704-911-5. pp 297-316.

ARANGO, L.; ESCOBAR, D. \& MONSALVE, E. (2013). Subempleo por ingresos y funcionamiento del mercado de trabajo en Colombia. Desarrollo y Sociedad, Issue 72, p157-203. 47p. ISSN 01203584, DOI 10.13043/DYS.72.4. Colombia.

AZEVEDO, V. \& BOUILLON, C. (2009). Social mobility in Latin America: A review of existing evidence. Research Department Working Papers 689, BID. HN110.5 .A94 2009 305.5 A994----dc22.

BOTERO, C.; RENDÓN, L. \& HERRERA, A. (2012). La salud en Colombia ¿̇un sistema de salud o enfermedad?. Revista Médica de Risaralda Vol 18(2), pp 183-184, diciembre. Disponible en Dialnet. Recuperado en junio 29/2015.

CADAVID, G. (2007). Estrategias de afrontamiento que utilizan las familias en proceso de separación con jóvenes de 12 a 20 años. Trabajo de Maestría en Psicología. Fundación Universidad del Norte. Barranquilla, Colombia.

CAQUEO, A. \& LEMOS, S. (2008). Calidad de vida y funcionamiento familiar de pacientes con esquizofrenia en una comunidad latinoamericana. Psicothema Vol. 20(4):577-582. ISSN 0214 - 9915.

CASTAÑO-DUQUE, G. \& GARCÍA-SERNA, L. (2012). Una revisión teórica de la calidad de la educación superior en el contexto colombiano. Educ. Educ. Vol. 15, No. 2, 219-243. Mayo-agosto. ISSN 0123-1294. Disponible en Ebsco-Host. Consultado en marzo 30/15.

CERDA, P.; CERDA, E. \& RODRÍGUEZ, L. (2009). Violencia y familia en Nuevo León. Journal Universidad Autónoma de Nuevo León (Ed). Vol 11 (2008): 1. ISSN 1405-9177. Disponible en E-Brary. Recuperado en junio 29/2015.

CUNHA, A.; RELVAS, A. \& SOARES, I. (2009). Anorexia nervosa and family relationships: Perceived family functioning, coping strategies, beliefs, and attachment to parents and peers. International Journal of Clinical and Health Psychology ISSN 1697-2600 Vol. 9(2): 229-240. Disponible en http:// www.redalyc.org/pdf/337/33712028003.pdf Consultado en noviembre 5/2014.

DE CARLO S. \& WADSWORTH, M. (2009). Coping with family conflict: What's helpful and what's not for low-income adolescents. J Child Fam Stud Vol. 18:192-202. DOI 10.1007/s10826008-9219-9. Disponible en base de datos Ebsco Host; Springer.

EATON, P.; DAVIS, B.; HAMMOND, P.; CONDON, E. \& MCGEE, Z. (2011). Coping strategies of family members of hospitalized psychiatric patients. Hindawi Publishing Corporation Nursing 
Research and Practice Volume 2011, Article ID 392705, 12 pages:1-12. Doi:10.1155/2011/392705.

FIDAN, T.; CEYHUN, H. \& KIRPINAR, I. (2011). Coping strategies and family functionality in youths with or without suicide attempts. Archives of Neuropsychiatry, published by Galenos. Doi: 10.4274/Npa.y5785. Disponible en base de datos Ebsco Host. Consultado en octubre 28/2014.

FURLANI, M. (2009). "El estado de bienestar: Auge y resquebrajamiento" en Revista Electrónica de Psicología Política, Año 7 No. 20. Julio - agosto.

GALVIS, L. \& MEISEL, A. (2014). Aspectos regionales de la movilidad social y la igualdad de oportunidades en Colombia. Documentos de trabajo sobre economía regional Banco de la República, Centro de Estudios Económicos Regionales, Cartagena, Núm 196, enero. ISSN 16923715. Disponible en http://www.banrep.gov.co/docum/Lectura_finanzas/pdf/dtser_196.pdf. Consultado en marzo 28/15.

GARRANZO, R. \& GÓMEZ L. (2006). La reconstrucción de las sociedades post-conflicto: Guatemala después de los Acuerdos de Paz. Quorum Revista de Pensamiento Iberoamericano, ISSN 1575-4227, No 14, 2006, págs. 161-177. Disponible en Dialnet Plus. Recuperado en junio 25/2015.

GARCÍA, E. (2012). ¿Es Colombia un estado corrupto? La corrupción como problema jurídico y como estado sociológico-moral. Una reflexión sobre el presente de Colombia en el tiempo de los "eveilleurs" (los desmitificadores de sueños). Vniversitas N" 125: 187-217, julio-diciembre. Bogotá, Colombia. Disponible en Ebsco Host. Consultado en marzo 31/15.

GARZÓN, J. C. (2003). Las limitaciones de la paz. Revista de Estudios Sociales N" 15: 125-132, junio. Bogotá, Colombia. ISSN-e 0123-885X. Disponible en Dialnet plus. Consultado en junio 25/15.

GERSON, R.; WONG, C.; DAVIDSON, L.; MALASPINA, D.; MCGLASHAN, Th. \& OTROS (2011). Self-reported coping strategies in families of patients in early stages of psychotic disorder: an exploratory study. Early intervention in psychiatry. Vol 5:76-80. doi:10.1111/j.17517893.2010.00251.x. Disponible en base de datos Ebsco Host. Consultado en octubre 24/2014.

GREY, D. (2006). Coping over time: the parents of children with autism. Journal of Intellectual Disability Research, Vol 50(12):970-976, december. Doi: 10.1111/j.1365-2788.2006.00933. Australia. Disponible en base de datos Ebsco Host http://ezproxy.unicartagena.edu.co:2150/ ehost/pdfviewer/pdfviewer?sid=ec7b654d-c536-41b0-b07f-8290d46a2145\%40sessionmgr115 \&vid $=4$ \&hid $=116$. Consultado en noviembre 5/2014

JiMÉNEZ, M.; AMARÍS, M., \& VALLE, M. (2012). Afrontamiento en crisis familiares: El caso del divorcio cuando se tienen hijos adolescentes. Salud Uninorte Vol.28(1): 99-112. Barranquilla, Colombia.

KARTALOVA-O'DOHERTY, Y. \& TEDSTONE, D. (2008). Coping strategies and styles of family carers of persons with enduring mental illness: a mixed methods analysis. Health Research Board (HRB) copyright. Journal compilation Nordic College of Caring Science (22):19-28. Disponible en http://ezproxy.unicartagena.edu.co:2150/ehost/pdfviewer/pdfviewer?sid=ec7b654d-c536-41b0b07f-8290d46a2145\%40sessionmgr115\&vid=7\&hid=116 consultado en noviembre 05/2014.

KISER, L.; DONOHUE, A.; MEDOFF, D. \& BLACK, M. (2010). Strengthening Family Coping Resources: The Feasibility of a Multifamily Group intervention for Families Exposed to Trauma. 
Journal of Traumatic Stress, Vol. 23(6): 802-806 December. University of Maryland School of Medicine. Disponible en wileyonlinelibrary.com DOI: 10.1002/jts.20587. Consultado en noviembre $01 / 2014$.

KRAMER, U. (2010) Coping and defence mechanisnns: What's the difference? - Second act. Psychology and Psychotherapy: Theory. Research and Practice (2010). 83. 207-221. Pág 207-221. Disponible en sitio http://ezproxy.uninorte.edu.co:4811/eds/pdfviewer/ pdfviewer?vid=3\&sid=e565341c-cc04-4472-92de-68fac3870de2\%40sessionmgr4003\&hid=4208. Recuperado en abril 26/2014.

FOLKMAN, S.; LAZARUS, R.; SCHETTER, C.; DELONGIS, A. \& GRUEN, R. (1986). Dynamics of a Stressful Encounter: Cognitive Appraisal, Coping, and Encounter Outcomes. Journal of Personality and Social Psychology Vol. 50 (5), 992-1003. Disponible en sitiofile:///D:/Mis\%20 documentos/1986\%20FolkmanLazarus_Dynamics\%20of\%20a.pdfConsultado en fecha junio $16 / 2015$

GARCÍA L., Ana \& RODRÍGUEZ R., J. Carlos (2005). Afrontamiento familiar ante la enfermedad mental. Revista Cultura de los Cuidados, Semestre II, Año IX No.18. pp 45-51.

IRIGOYEN-CORIA, A.; GÓMEZ-CLAVELINA, F.; TERÁN, M. \& PONCE-ROSAS, E. (1999). Pobreza y daños a la salud a nivel familiar. Archivos de medicina familiar 1(1):27-34. Disponible en Ebsco Host. México.

LEÓN, A. (2007). Qué es la educación. Educere Vol 11 núm 39, octubre-diciembre. Universidad de los Andes, Venezuela. Pp 595-64. Disponible en Redalyc, Red de Revistas Científicas de América Latina, el Caribe, España y Portugal. Consultado en marzo 30/15.

LOIGNON, C.; HUDON, C.; GOULET, E.; BOYER, S.; DE LAAT, M. et al (2015). Perceived barriers to healthcare for persons living in poverty in Quebec, Canada: the EQUIheal Th Y project. International Journal for Equity in Health Sample doi:10.1186/s12939-015-0135-5, January. Consultado en marzo 24/15.

McCUBBIN, H. I.; LARSEN, A. \& OLSON, D. H. (1981). FCOPES. Family crisis oriented personal evaluation scales. En D.H. Olson, H.I. McCubbin, H. Barnes, A. Larsen, M. Muxen y L.R. Wilson (Eds.), Family inventories. University of Minnesota, St. Paul

MANTILLA, S. C. (2009). "Hacia una perspectiva "glocal" del conflicto armado en Colombia: dinámicas y actores en los espacios transfronterizos y transnacionales" en Papel Político jul-dic2009, Vol. 14 Issue 2, p581-607. 27p.

MARTín, E.; FAJARDo, C.; GUTIÉRREZ, A. \& PALMA, D. (2007). Estrategias de afrontamiento de crisis causadas por desempleo en familias con hijos adolescentes en Bogotá. Acta Colombiana de Psicología Vol. 10(2). 127-141 julio-diciembre, Universidad Católica de Colombia. Disponible en: http://www.redalyc.org/articulo.oa?id=79810213 consultado en octubre 25/2014.

MARTÍNEZ-RODRÍGUEZ, J.; GARCÍA-CHONG, N.; TRUJILLO-OLIVERA, L. \& NORIEROESCALANTE, L. (2015). Inseguridad alimentaria y vulnerabilidad social en Chiapas: el rostro de 
la pobreza. Nutrición Hospitalaria. ene2015, Vol. 31 Issue 1, p475-481. 7p. Consultado en marzo $24 / 2015$

MELÉNDEZ, M. (2010). Estrategias de afrontamiento individual y familiar en personas desmovilizadas de los grupos armados en proceso de reintegración frente a la violencia intrafamiliar en la pareja. Trabajo de grado Maestría en Psicología. Fundación Universidad del Norte. Barranquilla, Colombia.

NAVIA, C. (2008). Afrontamiento familiar en situaciones de secuestro extorsivo económico. Revista latinoamericana de psicología. Vol. 40(1):59-72. Disponible en http://www.redalyc.org/ pdf/805/80500105.pdf Consultado en noviembre04/2014

NEWMAN, E. \& VAN-SELM, J. (2003). Refugees and forced displacement: International security, human vulnerability, and the state. United Nations University Press.ISBN 92-808-1086-3. Hong Kong.

OSHIO, T. \& KAN, M. (2014). Multimensional poverty and health: evidence from a nationwide survey in Japan. International Journal for Equity in Health 2014, 13:128 doi:10.1186/s12939014-0128-9. Consultado en marzo 24/15.

PURYEAR, J. \& MALLOY, M. (2009). Pobreza y desigualdad en América Latina. Política Social Síntesis No.1. The Inter-American Dialogue. Noviembre. Disponible en www.thedialogue.org Consultado en marzo 28/15.

RAVAZZOLA, M. (2001). Resiliencia familiares. En A. Melillo y E. Suárez (compiladores). Resiliencia, descubriendo las propias fortalezas. Paidós Tramas Sociales. Buenos Aires, Argentina.

REY de MARULANDA, N. \& GUZMÁN, J. (2003). "Inequidad, desarrollo humano y política social: importancia de las "condiciones iniciales" en Documentos de Trabajo I-51 Es, Banco Interamericano de Desarrollo, Departamento de Integración y Programas Regionales, junio, 42p.

RUANO, R. \& SERRA, E. (2000). Estrategias de afrontamiento en familias con hijos adolescentes. Anales de psicología, Vol. 16( 2):199-206

TURIZO, Y. (2012). Estrategias de afrontamiento familiar e inteligencias personales de jóvenes desplazados por la violencia sociopolítica radicados en la ciudad de Barranquilla. Trabajo de grado Maestría en Desarrollo Social. Fundación Universidad del Norte. Barranquilla, Colombia

VALENCIA, L. (2007). "Por qué confluyeron paramilitares y políticos? En Revista Arcanos Año 10/No.13. ISSN 0124-4159, marzo.

VÉLEZ, M. (2014). Educación universitaria como factor de movilidad social. Revista Telos mayoagosto, Vol 16(2): 207-225. ISSN 1317-0570. Base de datos Fuente Académica Premier. Recuperado en junio $24 / 2015$.

Universidad Nacional (2009). Claves para el debate público No.27, julio. Bogotá - Colombia. DANE, Encuesta Nacional de Calidad de Vida 2013 (2014) (ECV). Boletín de prnesa Año 2012-2013. Cód.: DIE-020-PD-01-r5_V.2 Fecha: 19-03-2014 Pág. 1. Marzo. Bogotá Colombia. 
OCDE. Estudios económicos de la OCDE, Colombia: Visión general.(2015). Disponible en sitio http://www.thedialogue.org/PublicationFiles/Politica\%20Social\%20Sintesis\%20No\%201\%20 Pobreza\%20y\%20Desigualdad\%20en\%20America\%20Latina.pdf Consultado en marzo 27/15.

MENDOZA-RAMOS, L. (2015). Correlación entre las estrategias de afrontamiento familiar y las redes sociales en familias en contexto de pobreza. Trabajo de grado para optar al título de Magíster en Desarrollo Social, Universidad del Norte, Barranquilla, no publicado. 

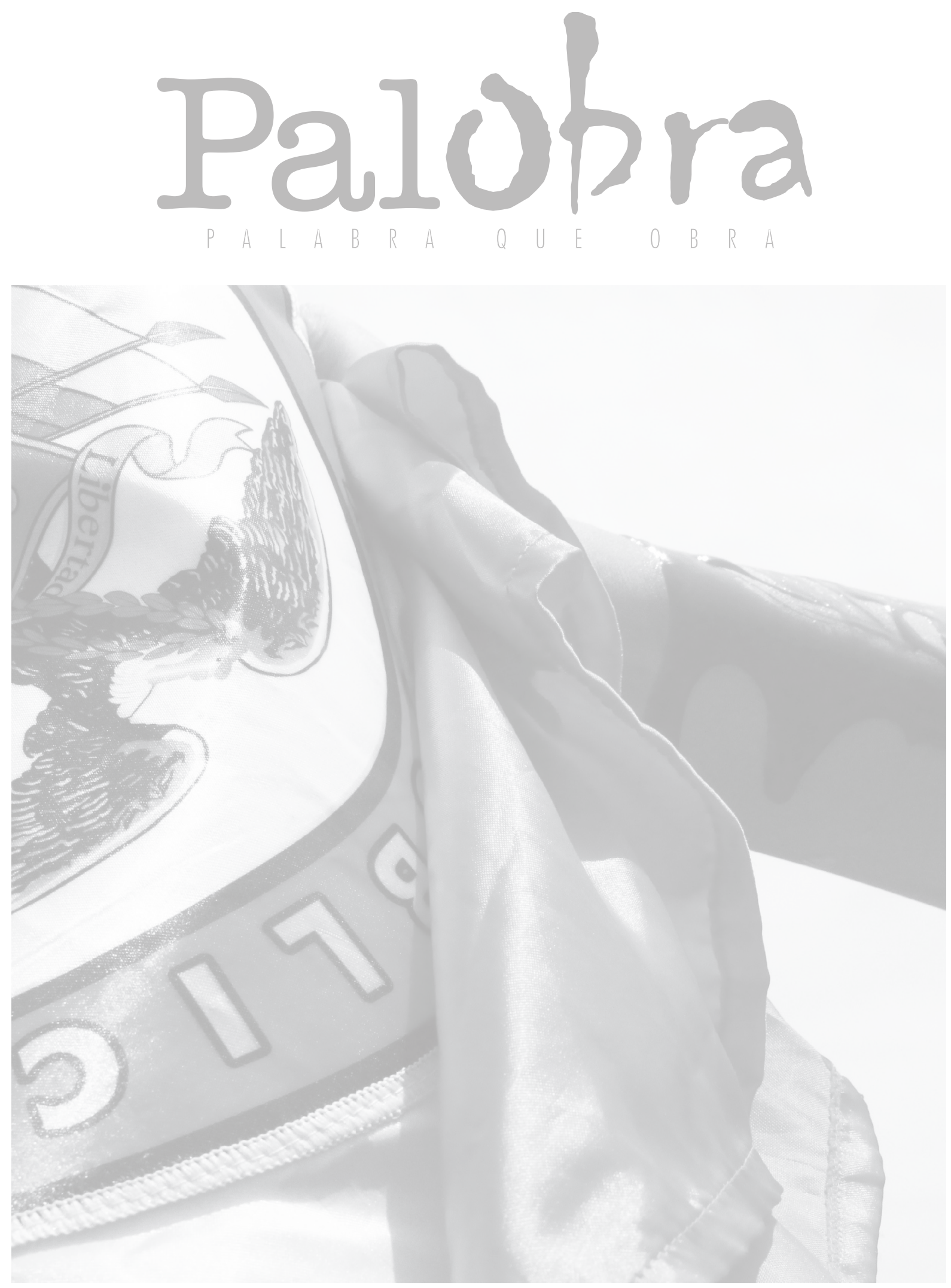

Foto: Andrés Silva 